\title{
Electrical stimulation by semi-implantable electrodes decreases the levels of proteins associated with sciatic nerve injury-induced muscle atrophy
}

\author{
JUN LIU, KUANGWEN LI, XIONGJIE HUANG, JUNFENG XIE and XINFENG HUANG
}

Department of Hand Surgery, the Affiliated Nanhua Hospital, University of South China, Hengyang, Hunan 421001, P.R. China

Received November 22, 2012; Accepted April 11, 2013

DOI: $10.3892 / \mathrm{mmr} .2013 .1487$

\begin{abstract}
Muscle atrophy is a disease that is usually caused by denervation. The aim of the present study was to determine whether electrical stimulation by semi-implantable electrodes is capable of decreasing the levels of specific proteins associated with sciatic nerve injury-induced muscle atrophy. Male Sprague Dawley (SD) rats with damaged sciatic nerves were maintained on a 12-h light/dark cycle. Thirty-two SD rats were randomly allocated into 4 groups (each group, $n=8$ ). The rats in group $\mathrm{C}$ received no electrical stimulation; the rats in groups $\mathrm{D}$, $\mathrm{N}$ and DN received electrical stimulation by semi-implantable electrodes during the daytime alone, nighttime alone and both the daytime and nighttime, respectively. Immunoblot assays were performed to detect the expression of cellular proteins associated with muscle atrophy. The number of muscle satellite cells was determined using a microscope, indicating that electrical stimulation increased the number of muscle satellite cells. Immunoblot assay results showed that electrical stimulation reduced the expression levels of cathepsin L, calpain 1 and the ubiquitinated muscle ring finger-1 (MuRF-1) protein. In conclusion, electrical stimulation by semi-implantable electrodes constitutes a potential method for the treatment of sciatic nerve injury-induced muscle atrophy. The decreased expression levels of the cellular proteins cathepsin L and calpain 1, as well as the ubiquitinated protein MuRF-1, are associated with the attenuation of sciatic nerve injury-induced muscle atrophy.
\end{abstract}

\section{Introduction}

Muscle atrophy is a disease that may be caused by denervation $(1,2)$, joint immobilization $(3,4)$, hindlimb unloading $(5,6)$ and spinal cord injury (7-9). Resistance exercise training is

Correspondence to: Dr Jun Liu, Department of Hand Surgery, the Affiliated Nanhua Hospital, University of South China, No. 336 Dongfeng South Road, Hengyang, Hunan 421001, P.R. China E-mail: liujun88665@163.com

Key words: electrical stimulation, muscle atrophy, cathepsin L, calpain 1, muscle ring finger-1 a straightforward therapeutic method that is used to prevent muscle atrophy. In particular, isometric contraction exercise has significant protective effects against muscle atrophy since it is a high-intensity activity compared with other types of exercise $(10,11)$. However, exercise training is not suitable for patients with severe disease or who are unable to perform voluntary limb movements. Therefore, electrical stimulation has also been used in the treatment of patients with muscle atrophy in a clinical setting (22).

Electrical stimulation includes transcutaneous electrical stimulation, as well as electrical stimulation by implantable and semi-implantable electrodes. Transcutaneous electrical stimulation is straightforward and easy to perform. However, the stimulation is attenuated by the skin, which may result in the diffusion of signals. Electrical stimulation by implantable electrodes is characterized by precise stimulation; however, the electrodes have to be implanted into the muscles and eventually removed. Furthermore, stimulation cannot be easily adjusted since the electrodes are implanted. With regard to electrical stimulation by semi-implantable electrodes, the electrodes are implanted into the muscles; however, they remain linked to the equipment, which enables the intensity of the stimulation to be easily adjusted. The effectiveness of electrical stimulation depends on the intensity of the current, which is affected by the frequency $(12,13)$, number of contractions $(1,14)$ and chronaxie $(2)$.

Electrical stimulation attenuates the decrease in muscle mass and muscle fiber cross-sectional area (15). Three major protein degradation pathways have been reported to be involved in such muscle atrophy-associated alterations (16): i) The ubiquitin-proteasome pathway, which includes conjugation of multi-ubiquitin moieties to the substrate and degradation of the tagged protein by the $26 \mathrm{~S}$ proteasome (17). The muscle-specific ubiquitin ligases, including atrogin-1/MAFbx (atrogin-1) and muscle ring finger-1 (MuRF-1) are overexpressed in atrophied muscles (16); ii) the lysosomal protease pathway involving cathepsins, which have been shown to increase activity in atrophied muscles (18) and iii) the calpain pathway involving calpain 1 and calpain 2, which are cytosolic calcium-dependent proteases with an increased expression in atrophied muscles (18).

Examination of the electrical stimulation-induced alterations in the expression levels of factors involved in the 
above-mentioned pathways may improve our understanding of therapeutic approaches to muscle atrophy. In the present study, we established Sprague Dawley (SD) rat models with damaged sciatic nerves. Electrical stimulation by semi-implantable electrodes was administered to these SD rat models during the daytime alone, nighttime alone and both the daytime and nighttime. Evaluation of the muscle wet weight and the number of muscle satellite cells, as well as western blot analysis was performed. Electrical stimulation was demonstrated to reduce the expression levels of the cellular proteins that contribute to muscle atrophy, including cathepsin L, calpain 1 and the ubiquitinated MuRF-1 protein.

\section{Materials and methods}

Experimental groups. Male SD rats with damaged sciatic nerves (age, 46-49 days old; weight, 200-225 g) were maintained in a 12-h light/dark cycle. Thirty-two rats were randomly allocated into 4 groups (each group, $\mathrm{n}=8$ ) and housed individually in standard wire mesh cages. Food and sterile tap water were made freely available. The rats received electrical stimulation by semi-implantable electrodes during the daytime alone (group D), nighttime alone (group N) or during the daytime and nighttime (group DN). The rats in group $\mathrm{C}$ received no electrical stimulation and served as the control group. All of the experiments in this study were approved by the Animal Care Committee of the Affiliated Nanhua Hospital, University of South China (Hengyang, Hunan, China).

Electrical stimulation. The electrical stimulation equipment was provided by the South China Hospital Affiliated to the University of South China.

The male SD rats were anesthetized. The sciatic nerve was sharply cut at a site $5 \mathrm{~mm}$ from the lower edge of the piriformis. Under a microscope, by performing epineurium suture with a non-invasive suture needle, one end of an insulated wire was implanted into the proximal sciatic nerve. The middle part of the wire was fixed on the starting point of the iliac muscle tendon. The other end of the wire was left outside of the skin. The wire was then connected to the electrical stimulation equipment, which was modulated to the correct parameters. The continuous electrical stimulation parameters used were as follows: voltage, $1.5 \mathrm{~V}$; frequency, $50 \mathrm{~Hz}$; pulse width, $0.5 \mathrm{~ms}$ and stimulus interval, $1 / 4,1 / 3$ or $1 / 2 \mathrm{sec}$. The same stimulation parameters were used for each of the 3 groups (D, N and DN) in which the rats received electrical stimulation.

Sample preparation and histological analysis. Twelve hours following the last stimulation, all of the animals were anesthetized by an intraperitoneal injection of sodium pentobarbital, and then the gastrocnemius muscle was removed and weighed using an electronic balance. The animals were then sacrificed by an overdose of sodium pentobarbital. Isolated parts from the muscle sample $(\sim 10 \mathrm{mg})$ were immediately frozen in acetone, cooled in dry ice, and maintained at $-80^{\circ} \mathrm{C}$ for further histological examination and western blot analysis. Serial sections were cut from the middle part of the muscle belly in the gastrocnemius muscle and stained for lead and uranium. The number of satellite cells was determined with transmission electron microscopy.
Western blot analysis. The total protein was harvested from muscle tissues, separated on 10\% SDS-PAGE gel and then examined by immunoblot analysis. The primary antibodies against cathepsin $\mathrm{L}(\sim 38 \mathrm{kDa})$, calpain 1 (large units, $\sim 80 \mathrm{kDa})$, MuRF-1 ( $44 \mathrm{kDa})$ and $\beta$-actin were purchased from Santa Cruz Biotechnology, Inc. [Santa Cruz, CA, USA; anti-cathepsin L (H-80), cat. no. sc-10778, 1:200; anti-calpain 1 (D-11), cat. no. sc-271313, 1:200; anti-MuRF1 (SW-53), cat. no. sc-134397; anti- $\beta$-actin, cat. no. sc-130301, 1:10,000]. The secondary antibodies used in this study were goat anti-mouse IgG-horseradish peroxidase (HRP; cat. no. sc-2005, 1:10,000; Santa Cruz Biotechnology, Inc.) and goat anti-rabbit IgG-HRP (cat. no. sc-2004, 1:5,000; Santa Cruz Biotechnology, Inc.). Bound antibodies were detected using the ECL system (Pierce Biotechnology, Inc., Rockford, IL, USA). The immunoblot assays were repeated at least 3 times. The mean normalized optical density (OD) of the cathepsin L, calpain 1 and MuRF-1 protein bands relative to the OD of the $\beta$-actin band from the same animal was calculated.

Statistical analysis. The experimental data are expressed as the means \pm standard error of the mean (SEM). $\mathrm{P}<0.05$ was considered to indicate a statistically significant difference.

\section{Results}

Electrical stimulation increases muscle wet weight. Thirty-two SD rats were randomly allocated into 4 groups (each group, $\mathrm{n}=8$ ). The rats in group $\mathrm{C}$ received no electrical stimulation; the rats in groups $\mathrm{D}, \mathrm{N}$ and $\mathrm{DN}$ received electrical stimulation by semi-implantable electrodes during the daytime alone, nighttime alone and both the daytime and nighttime, respectively. The values of body weight and the gastrocnemius muscle wet weight are shown in Table I. The values of body weight in the 4 groups were similar, without statistical differences. The mean value of the gastrocnemius muscle wet weight in groups D, N and DN were significantly increased compared with that in group $\mathrm{C}(\mathrm{P}<0.05)$. The mean value of muscle wet weight in group DN was significantly increased compared with those in groups $\mathrm{D}$ and $\mathrm{N}(\mathrm{P}<0.05)$, although there was no significant difference between groups $\mathrm{D}$ and $\mathrm{N}$.

The mean value of the ratio of wet muscle weight to body weight in group DN was also significantly increased compared with the ratio in groups $\mathrm{D}$ and $\mathrm{N}(\mathrm{P}<0.05)$. These results suggest that electrical stimulation increases the muscle wet weight.

Electrical stimulation increases the number of muscle satellite cells. In the experiments described above using electrical stimulation by semi-implantable electrodes, muscle satellite cells were isolated from the rats in each of the 4 groups. The number of muscle satellite cells was counted using a microscope. The mean number of muscle satellite cells and the ratio of the cell number to body weight are shown in Table II. The number of muscle satellite cells in groups D, N and DN was significantly increased compared with that in group $\mathrm{C}$ $(\mathrm{P}<0.05)$. The number of muscle satellite cells in group DN was significantly increased compared with that in groups $\mathrm{D}$ and $\mathrm{N}$ $(\mathrm{P}<0.05)$, although there was no significant difference between the number of satellite cells in groups D and $\mathrm{N}$. The ratio of cell number to body weight in group DN was also significantly 
Table I. Body weight, wet weight of the gastrocnemius muscle and the wet muscle weight/body weight ratio of all groups $($ mean \pm SEM).

\begin{tabular}{lcccc}
\hline & \multicolumn{3}{c}{ Group (n=8) } \\
\cline { 2 - 5 } Weight & $\mathrm{C}$ & $\mathrm{D}$ & $\mathrm{N}$ & $\mathrm{DN}$ \\
\hline Muscle wet weight (mg) & $329.2 \pm 6.8$ & $411.5 \pm 4.6^{\mathrm{a}}$ & $428.8 \pm 2.9^{\mathrm{a}}$ & $492.3 \pm 10.2^{\mathrm{a}, \mathrm{b}}$ \\
Body weight (g) & $218.1 \pm 4.2$ & $216.3 \pm 4.1$ & $212.4 \pm 3.9$ & $215.3 \pm 3.8$ \\
Muscle wet weight/body weight (mg/g) & $1.54 \pm 0.02$ & $1.91 \pm 0.03^{\mathrm{a}}$ & $2.02 \pm 0.01^{\mathrm{a}}$ & $2.31 \pm 0.01^{\mathrm{a}, \mathrm{b}}$ \\
\hline
\end{tabular}

Group C, rats received no semi-implantable electrical stimulation; group D, rats received semi-implantable electrical stimulation during the daytime; group $\mathrm{N}$, rats received semi-implantable electrical stimulation during the nighttime; group DN, rats received semi-implantable electrical stimulation during the daytime and nighttime. ${ }^{a} \mathrm{P}<0.05$ compared with group $\mathrm{C}$; ${ }^{\mathrm{P}} \mathrm{P}<0.05$ compared with groups $\mathrm{D}$ and $\mathrm{N}$.

Table II. Numbers of muscle satellite cells in the cross-sectional area and ratios of cell number to body weight in all the groups $($ mean \pm SEM) .

\begin{tabular}{lcccc}
\hline & \multicolumn{3}{c}{ Group $(\mathrm{n}=8)$} \\
\cline { 2 - 5 } Characteristic & $\mathrm{C}$ & $\mathrm{D}$ & $\mathrm{N}$ & $\mathrm{DN}$ \\
\hline Number of muscle satellite cells $\left(\mu \mathrm{m}^{2}\right)$ & $112 \pm 19$ & $326 \pm 34^{\mathrm{a}}$ & $402 \pm 53^{\mathrm{a}}$ & $823 \pm 91^{\mathrm{a}, \mathrm{b}}$ \\
Body weight $(\mathrm{g})$ & $218.1 \pm 4.2$ & $216.3 \pm 4.1$ & $212.4 \pm 3.9$ & $215.3 \pm 3.8$ \\
Number of muscle satellite cells/body weight $\left(\mu \mathrm{m}^{2} / \mathrm{g}\right)$ & $0.52 \pm 0.01$ & $1.51 \pm 0.03^{\mathrm{a}}$ & $1.90 \pm 0.03^{\mathrm{a}}$ & $3.83 \pm 0.05^{\mathrm{a}, \mathrm{b}}$ \\
\hline
\end{tabular}

${ }^{\mathrm{a}} \mathrm{P}<0.05$ compared with group $\mathrm{C}$; ${ }^{\text {}} \mathrm{P}<0.05$ compared with groups $\mathrm{C}$ and $\mathrm{N}$.

A

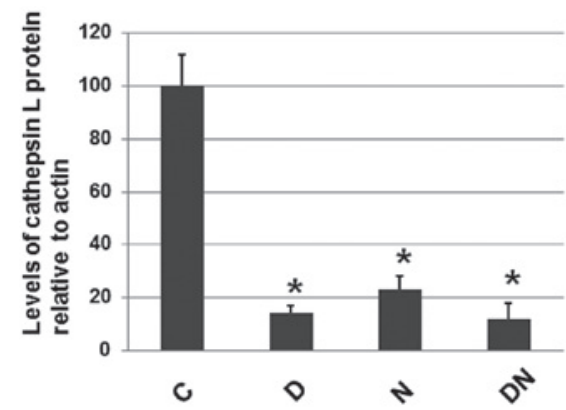

B

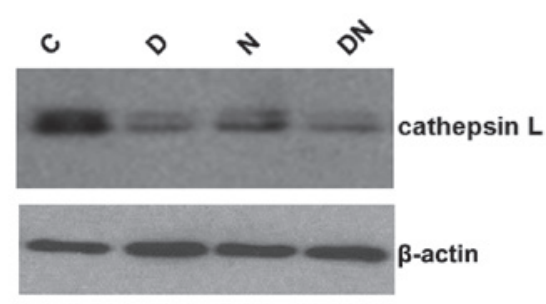

Figure 1. Immunoblot analysis of cathepsin $\mathrm{L}$ in the rats of all 4 groups. The rats in group $\mathrm{C}$ received no electrical stimulation; the rats in groups $\mathrm{D}, \mathrm{N}$ and DN received electrical stimulation by semi-implantable electrodes during the daytime, nighttime and both the daytime and nighttime, respectively. (A) Total protein was extracted from the muscle tissue of rats, separated on an SDS-PAGE gel and examined by immunoblot analysis. Primary antibodies against cathepsin $\mathrm{L}$ and $\beta$-actin were used. The secondary antibody used was goat anti-rabbit IgG-HRP (cat. no. sc-2004, 1:5,000). Bound antibodies were detected using the ECL system. The cathepsin L protein was $\sim 38 \mathrm{kDa}$. Histograms show the mean normalized OD of the cathepsin L protein bands relative to the OD of the $\beta$-actin band from the rats in each group. Error bars show the SEM. ${ }^{*} \mathrm{P}<0.05$ compared with the cathepsin $\mathrm{L}$ protein levels in the rats of group C. (B) Representative blots. HRP, horseradish peroxidase; OD, optical density; SEM, standard error of the mean. increased compared with those in groups $\mathrm{D}$ and $\mathrm{N}(\mathrm{P}<0.05)$, although there was no significant difference between the ratios in groups D and N. These results suggest that electrical stimulation increases the number of muscle satellite cells.

Electrical stimulation reduces the level of cathepsin L. To investigate whether electrical stimulation by semi-implantable electrodes affects the expression levels of cathepsin L, a protein that is often expressed at elevated levels in atrophied muscles, the total protein was extracted from the muscle tissue of rats from all 4 groups. The expression level of cathepsin $\mathrm{L}$ was determined by western blot analysis, with cellular $\beta$-actin serving as a loading control. The mean normalized $\mathrm{OD}$ of the cathepsin $\mathrm{L}$ protein bands relative to the OD of the $\beta$-actin band from each group was calculated and subjected to statistical analysis. The error bars show the SEM ( $\mathrm{P}<0.05$; Fig. 1A). Representative blots are shown in Fig. 1B.

As shown in Fig. 1, the expression levels of cathepsin $L$ in the rats of groups $\mathrm{D}, \mathrm{N}$ and $\mathrm{DN}$ were significantly decreased compared with group $\mathrm{C}$, which received no electrical stimulation. However, the cathepsin L expression levels in groups D, $\mathrm{N}$ and DN were similar. These results suggest that electrical stimulation by semi-implantable electrodes during the daytime, nighttime or both the daytime and nighttime decreases the expression levels of cathepsin L.

Electrical stimulation reduces the expression levels of calpain 1. To investigate whether electrical stimulation by 
A

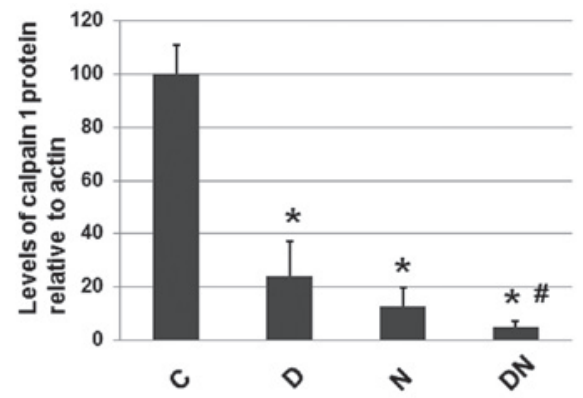

B

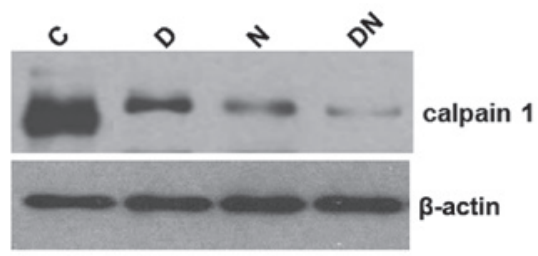

Figure 2. Immunoblot analysis of calpain 1 in the rats of all 4 groups. The rats in group $\mathrm{C}$ received no electrical stimulation; the rats in groups $\mathrm{D}, \mathrm{N}$ and DN received electrical stimulation by semi-implantable electrodes during the daytime, the nighttime and both the daytime and nighttime, respectively. (A) Total protein was extracted from the muscle tissue of rats, separated on an SDS-PAGE gel and subjected to immunoblot analysis. Primary antibodies against calpain 1 and $\beta$-actin were used. The secondary antibody used was goat anti-mouse IgG-HRP (cat. no. sc-2005, 1:10,000). Bound antibodies were detected using the ECL system. The calpain 1 protein was $\sim 80 \mathrm{kDa}$. Histograms show the mean normalized OD of the calpain 1 protein bands relative to the $\mathrm{OD}$ of the $\beta$-actin band from the rats in each group. Error bars show the SEM. " $\mathrm{P}<0.05$ compared with the calpain 1 protein levels in the rats of group $\mathrm{C} ;{ }^{*} \mathrm{P}<0.05$ compared with the calpain 1 protein levels in the rats of groups D and N. (B) Representative blots. HRP, horseradish peroxidase; OD, optical density; SEM, standard error of the mean; MuRF-1, muscle ring finger-1.

semi-implantable electrodes affects the expression levels of calpain 1, the total protein was extracted from the muscle tissue of rats from each group. The expression levels of calpain 1 were determined by western blot analysis, with the cellular $\beta$-actin protein serving as a loading control. The mean normalized OD of the calpain 1 protein bands relative to the OD of the $\beta$-actin band from each group was calculated and subjected to statistical analysis. Error bars show the SEM $(\mathrm{P}<0.05$; Fig. 2A). Representative blots were shown in Fig. 2B.

As shown in Fig. 2, the expression levels of calpain 1 in the rats of the groups which received electrical stimulation (D, $\mathrm{N}$ and $\mathrm{DN}$ ) by semi-implantable electrodes were significantly decreased compared with the expression levels in the rats of group C, which received no electrical stimulation. Furthermore, the calpain 1 expression levels in the rats of group DN were lower compared with those in the rats of groups $\mathrm{D}$ and $\mathrm{N}$. These results suggest that electrical stimulation by semi-implantable electrodes during the daytime, nighttime and both the daytime and nighttime decreases the levels of calpain 1. Additionally, electrical stimulation during the daytime and nighttime was more effective compared with electrical stimulation during the daytime and nighttime alone.

Electrical stimulation decreases the levels of the ubiquitinated MuRF-1 protein. To investigate whether electrical stimulation by semi-implantable electrodes affects the expression levels of MuRF-1, a protein that is often elevated
A

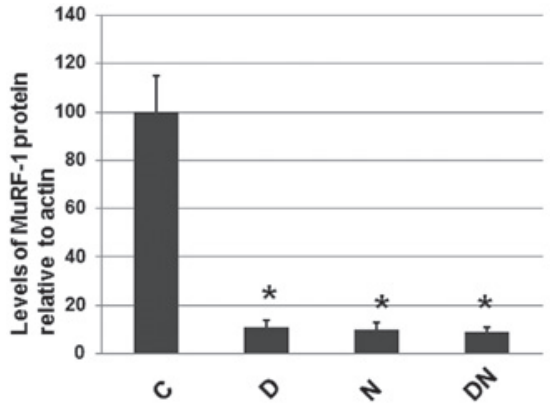

B

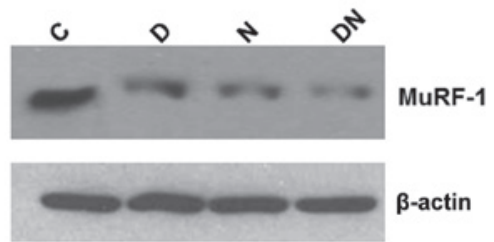

Figure 3. Immunoblot analysis of MuRF-1 in the rats of all 4 groups. The rats in group $\mathrm{C}$ received no electrical stimulation; the rats in groups $\mathrm{D}, \mathrm{N}$ and DN received electrical stimulation by semi-implantable electrodes during the daytime, the nighttime and both the daytime and nighttime, respectively. (A) Total protein was extracted from the muscle tissue of rats, separated on an SDS-PAGE gel and subjected to immunoblot analysis. Primary antibodies against MuRF-1 and $\beta$-actin were used. The secondary antibody used was goat anti-mouse IgG-HRP (cat no. sc-2005, 1:10,000). Bound antibodies were detected using the ECL system. The MuRF-1 protein was $\sim 44 \mathrm{kDa}$. Histograms show the mean normalized OD of the MuRF-1 protein bands relative to the OD of the $\beta$-actin band from the rats in each group. Error bars show the SEM. ${ }^{*} \mathrm{P}<0.05$ compared with the MuRF-1 protein levels in the rats of group C. (B) Representative blots. HRP, horseradish peroxidase; OD, optical density; SEM, standard error of the mean; MuRF-1, muscle ring finger-1.

in atrophied muscles, the total protein was extracted from the muscle tissue of rats from all 4 groups. The expression levels of MuRF-1 were determined by western blot analysis, with the cellular $\beta$-actin protein serving as a loading control. The mean normalized OD of the MuRF-1 protein bands relative to the OD of the $\beta$-actin band from rats in each group was calculated and subjected to statistical analysis. Error bars show the SEM $(\mathrm{P}<0.05$; Fig. 3A). Representative blots are shown in Fig. 3B.

As shown in Fig. 3, the expression levels of MuRF-1 in the rats of all groups receiving electrical stimulation by semi-implantable electrodes during the daytime (group D), nighttime (group $\mathrm{N}$ ) or both the daytime and nighttime (group DN) were significantly decreased compared with the rats of group $\mathrm{C}$, which received no electrical stimulation. However, the MuRF-1 expression levels among groups D, $\mathrm{N}$ and DN were similar. These results suggest that electrical stimulation by semi-implantable electrodes during the daytime, nighttime or both the daytime and nighttime decreases the levels of MuRF-1.

\section{Discussion}

Muscle atrophy is a disease that is usually caused by denervation $(1,2)$, joint immobilization $(3,4)$, hindlimb unloading $(5,6)$ and spinal cord injury (7-9). Recently, several studies have reported that electrical stimulation may be used in the treatment of muscle atrophy-related diseases (19-24). In particular, 
neuromuscular electrical stimulation has been shown to increase muscle protein synthesis in elderly type 2 diabetic male patients (22). Electrical stimulation provides a degree of protection against the degeneration of target and non-target muscles during treatment with botulinum toxin type $\mathrm{A}$, a treatment modality commonly used in various neuromuscular disorders (19).

In the present study, male SD rats were randomly allocated into 4 groups. Depending on the group, the rats received either no electrical stimulation (group C) or electrical simulation by semi-implantable electrodes during the daytime (group D), nighttime (group $\mathrm{N}$ ) or during the daytime and nighttime (group DN). The body weight and the gastrocnemius muscle wet weight of the rats were measured. The number of muscle satellite cells was also detected using a microscope. Electrical stimulation was demonstrated to increase the number of muscle satellite cells. Electrical stimulation during the daytime and nighttime was demonstrated to be more effective compared with the other treatment strategies.

Stimulation cannot be easily adjusted when the electrodes are implanted. With regard to electrical stimulation by semi-implantable electrodes, the electrodes are implanted into the muscles; however, they remain linked to the equipment, allowing the intensity of the stimulation to be more easily adjusted. The effectiveness of electrical stimulation is determined by the intensity of the current, which is affected by the frequency $(12,13)$, number of contractions $(1,14)$ and chronaxie (2). To investigate the effect of electrical stimulation at the molecular level, the expression levels of three cellular proteins involved in the muscle atrophy process were determined. Immunoblot assay results indicated that electrical stimulation significantly reduces the expression levels of cathepsin L, calpain 1 and ubiquitinated protein MuRF-1.

Although the expression levels of cathepsin L, calpain 1 and ubiquitinated MuRF-1 protein were decreased by electrical stimulation compared with the expression levels in the rats of group $\mathrm{C}$, which received no electrical stimulation, only calpain 1 expression levels in group DN were lower compared with those in groups $\mathrm{D}$ and $\mathrm{N}$. These results indicate that the expression levels of other unknown cellular proteins may also be altered due to electrical stimulation.

Taken together, electrical stimulation by semi-implantable electrodes may constitute a potential method for the treatment of sciatic nerve injury-induced muscle atrophy. The decreased expression levels of the cellular proteins cathepsin $\mathrm{L}$ and calpain 1, as well as the ubiquitinated MuRF-1 protein are associated with the attenuation of sciatic nerve injury-induced muscle atrophy.

\section{References}

1. Dow DE, Cederna PS, Hassett CA, Kostrominova TY, Faulkner JA and Dennis RG: Number of contractions to maintain mass and force of a denervated rat muscle. Muscle Nerve 30: 77-86, 2004.

2. Russo TL, Peviani SM, Freria CM, Gigo-Benato D, Geuna S and Salvini TF: Electrical stimulation based on chronaxie reduces atrogin-1 and myoD gene expressions in denervated rat muscle Muscle Nerve 35: 87-97, 2007.

3. Fujita N, Fujimoto T, Tasaki H, Arakawa T, Matsubara T and Miki A: Influence of muscle length on muscle atrophy in the mouse tibialis anterior and soleus muscles. Biomed Res 30: 39-45, 2009.
4. Fujita N, Arakawa T, Matsubara T, Ando H and Miki A: Influence of fixed muscle length and contractile properties on atrophy and subsequent recovery in the rat soleus and plantaris muscles. Arch Histol Cytol 72: 151-163, 2009.

5. Fujino H, Ishihara A, Murakami S, et al: Protective effects of exercise preconditioning on hindlimb unloading-induced atrophy of rat soleus muscle. Acta Physiol (Oxf) 197: 65-74, 2009.

6. Takeda I, Fujino H, Murakami S, Kondo H, Nagatomo F and Ishihara A: Thermal preconditioning prevents fiber type transformation of the unloading induced-atrophied muscle in rats. J Muscle Res Cell Motil 30: 145-152, 2009.

7. Roy RR, Zhong H, Hodgson JA, Grossman EJ, Siengthai B, Talmadge RJ and Edgerton VR: Influences of electromechanical events in defining skeletal muscle properties. Muscle Nerve 26: 238-251, 2002.

8. Kim SJ, Roy RR, Zhong H, Suzuki H, et al: Electromechanical stimulation ameliorates inactivity-induced adaptations in the medial gastrocnemius of adult rats. J Appl Physiol 103: 195-205, 2007.

9. Kim SJ, Roy RR, Kim JA, Zhong H, Haddad F, Baldwin KM and Edgerton VR: Gene expression during inactivity-induced muscle atrophy: effects of brief bouts of a forceful contraction countermeasure. J Appl Physiol 105: 1246-1254, 2008.

10. Fitts RH: Effects of regular exercise training on skeletal muscle contractile function. Am J Phys Med Rehabil 82: 320-331, 2003.

11. Hurst JE and Fitts RH: Hindlimb unloading-induced muscle atrophy and loss of function: protective effect of isometric exercise. J Appl Physiol 95: 1405-1417, 2003.

12. Misawa A, Shimada Y, Matsunaga T and Sato K: The effects of therapeutic electric stimulation on acute muscle atrophy in rats after spinal cord injury. Arch Phys Med Rehabil 82: 1596-1603, 2001.

13. Boonyarom O, Kozuka N, Matsuyama K and Murakami S: Effect of electrical stimulation to prevent muscle atrophy on morphologic and histologic properties of hindlimb suspended rat hindlimb muscles. Am J Phys Med Rehabil 88: 719-726, 2009.

14. Dennis RG, Dow DE and Faulkner JA: An implantable device for stimulation of denervated muscles in rats. Med Eng Phys 25: 239-253, 2003.

15. Fujita N, Murakami S, Arakawa T, Miki A and Fujino H: The combined effect of electrical stimulation and resistance isometric contraction on muscle atrophy in rat tibialis anterior muscle. Bosn J Basic Med Sci 11: 74-79, 2011.

16. Jackman RW and Kandarian SC: The molecular basis of skeletal muscle atrophy. Am J Physiol Cell Physiol 287: C834-C843, 2004.

17. Glickman $\mathrm{MH}$ and Ciechanover A: The ubiquitin-proteasome proteolytic pathway: destruction for the sake of construction. Physiol Rev 82: 373-428, 2002.

18. Taillandier D, Aurousseau E, Meynial-Denis D, et al: Coordinate activation of lysosomal, $\mathrm{Ca}^{2+}$-activated and ATP-ubiquitindependent proteinases in the unweighted rat soleus muscle. Biochem J 316: 65-72, 1996.

19. Fortuna R, Horisberger M, Vaz MA, Van der Marel R and Herzog W: The effects of electrical stimulation exercise on muscles injected with botulinum toxin type-A (botox). J Biomech 46: 36-42, 2013.

20. Clair-Auger JM, Lagerquist O and Collins DF: Depression and recovery of reflex amplitude during electrical stimulation after spinal cord injury. Clin Neurophysiol 124: 723-731, 2013.

21. Lagerquist O, Mang CS and Collins DF: Changes in spinal but not cortical excitability following combined electrical stimulation of the tibial nerve and voluntary plantar-flexion. Exp Brain Res 222: 41-53, 2012.

22. Wall BT, Dirks ML, Verdijk LB, et al: Neuromuscular electrical stimulation increases muscle protein synthesis in elderly type 2 diabetic men. Am J Physiol Endocrinol Metab 303: E614-E623, 2012.

23. Huang J, Lu L, Zhang J, et al: Electrical stimulation to conductive scaffold promotes axonal regeneration and remyelination in a rat model of large nerve defect. PLoS One 7: e39526, 2012.

24. Santoro GA, Infantino A, Cancian L, Battistella G and Di Falco G: Sacral nerve stimulation for fecal incontinence related to external sphincter atrophy. Dis Colon Rectum 55: 797-805, 2012. 